\title{
Automated detection and analysis of volcanic thermal anomalies through the combined use of SEVIRI and MODIS
}

\author{
G. GANCI* and L. FORTUNA \\ Dipartimento di Ingegneria Elettrica Elettronica \\ e dei Sistemi, Universitá di Catania, Italy \\ *E-mail: ganci@ct.ingv.it \\ A. VICARI and C. DEL NEGRO \\ Istituto Nazionale di Geofisica e Vulcanologia \\ Sezione di Catania, Italy
}

\begin{abstract}
Multispectral infrared observations carried out by the spacecrafts have shown that spaceborne remote sensing of high-temperature volcanic features is feasible and robust enough to turn into volcano monitoring. Especially meteorological satellites have proven a powerful instrument to detect and monitor dynamic phenomena, such as volcanic processes, allowing very high temporal resolution despite of their low spatial resolution. An automated system that uses both EOS-MODIS and MSG-SEVIRI thermal satellite data was developed at the Istituto Nazionale di Geofisica e Vulcanologia of Catania for early hot spot detection and for estimating the temporal evolution of the average effusion rate during eruptive events. The advantage obtained by the use of both SEVIRI and MODIS data in increasing temporal coverage improving satellite monitoring of active volcanoes was also confirmed on Etna volcano during the early phase of 2008 eruption.
\end{abstract}

Keywords: remote sensing; Etna volcano; multi-platform.

\section{Introduction}

The timely and synoptic view afforded by satellite-based sensors provides an excellent means to monitor the thermal activity of a volcano and to estimate the effusion rate throughout an eruption $[2,4,9]$. In the past, data provided by Landsat with high spatial resolution and low temporal resolution (16 days) have been employed for the analysis of thermal analysis of active lava flows, lava domes, lava lakes and fumarole fields. Recently images with lower spatial but higher temporal resolution from meteorological satellites have been proved to be an ideal instrument for continuous moni- 
toring of volcanic activity, even though the relevant volcanic characteristics are much smaller than the nominal pixel size $\left(1-3 \mathrm{~km}^{2}\right)$. Indeed, despite of the fact that the volcanic features of interest are usually much smaller than the nominal pixel size of the sensors, meteorological satellites, such as the MODerate Resolution Imaging Spectro-radiometer (MODIS) and even the Spinning Enhanced Visible and Infrared Imager (SEVIRI), can detect emitted radiance in the shortwave infrared (SWIR) part of the electromagnetic spectrum, a region in which active lava flows, vents and domes emit copious amounts of energy. MODIS instrument offers up to 10 wavebands suitable for hot-spot detection, but provide data at least 4 times a day for any subaerial volcano with a spatial resolution of about $1 \mathrm{~km}$. In particular band 21, also known as the "fire channel", was designed to have a much higher saturation temperature of about $500 \mathrm{~K}$. For these reasons MODIS data were supported as basis for automated systems to detect and monitor volcanic eruptions for the entire globe. The launch of MSG SEVIRI on August 2002 provides a unique opportunity for a volcanic eruption detection system in real-time by providing images at 15 minutes interval. In spite of the low spatial resolution (3 $\mathrm{km}$ at nadir), the frequency of observations afforded by the MSG SEVIRI was recently applied both for fire detection [7] and for the monitoring of effusive volcanoes in Europe and Africa [6]. Since these two satellites show significantly different characteristics in spatial, spectral and temporal resolution, the aim of this work is to integrate the information coming from MODIS and SEVIRI data in order to obtain a better comprehension of the volcanic phenomenon. If near-real-time volcano monitoring is to be achieved using satellite data, images must be routinely received and analyzed rapidly. To this end, a multi-platform tool for satellite image analysis and volcanic processes characterization was developed. Computing routines were designed to allow for the joint exploitation of radiometers MODIS and SEVIRI in operational monitoring, in response to the need for fast and robust determination of hot spot detection and effusion rate estimation at active volcanoes. In this work, this monitoring system have been used to identify pixels covered by lava flow during the last Mt Etna eruption began in May 2008; then the hot spot pixels were processed in order to compute the time-varying discharge rates.

\section{The satellite technique}

The first step in the automatic system is the hotspot identification to locate possible thermal anomalies over satellite data. To this purpose, the contextual approach of Harris was implemented (i.e. the VAST code of [5]. As 
for MODIS images, this technique uses the difference between brightness temperature in channel 21 (or 22) and channel $31(\Delta T)$ and sets a $\Delta T$ threshold obtained from within the image, to define whether a pixel is hot. Due to the low $N E \Delta T$ of channel 22 , we use channel 22 data if they are unsaturated. If channel 22 is saturated, we use channel 21 . The algorithm first defines a "nonvolcanic" portion of the image and uses the maximum $\Delta T$ from that portion to set a threshold. Pixels belonging to the volcanic area are then scanned and all the pixels that are greater than the threshold are classified as hot. An example of the hot spot detection results is given in figure 1, where the observed flow field is superimposed to satellite image of 22 July showing good correspondence between the pixels flagged as hot and the actual lava extent. The same procedure was applied to SEVIRI data channel 4 and 9. A hotspot image during 15 May 2008 is showed in figure 2. The thermal methodology for obtaining effusion rates is based on $[1,8]$. It was adapted to satellite thermal data by [3], who proposed a method of estimating time-averaged discharge rates using measurements of the total thermal flux of active surface flows obtained using data from satellite based sensors [10]. Thermal analysis is carried out for each hot pixel located to give an estimate of the area and radiated heat flux of the thermal feature contained within the pixel. To achieve this we assume a three component thermal surface within the pixel and use the approach of [5], where reasonable bounds are placed on the temperature of the lava crust, cracks and ambient background, to obtain lava flow crust and crack area. We use three bands to solve the following nonlinear three-equation system:

$$
\left\{\begin{array}{l}
R_{x}=p_{b} L_{\lambda_{x}, T_{b}}+p_{c} L_{\lambda_{x}, T_{c}}+p_{h} L_{\lambda_{x}, T_{h}} \\
R_{y}=p_{b} L_{\lambda_{y}, T_{b}}+p_{c} L_{\lambda_{y}, T_{c}}+p_{h} L_{\lambda_{y}, T_{h}} \\
R_{z}=p_{b} L_{\lambda_{z}, T_{b}}+p_{c} L_{\lambda_{z}, T_{c}}+p_{h} L_{\lambda z, T_{h}}
\end{array}\right.
$$

Where $R_{x}, R_{y}, R_{z}$ are the corrected measured radiances in the channel x, $\mathrm{y}, \mathrm{z}$ (respectively channel $4[3.9 \mu \mathrm{m}]$, channel $9[10.8 \mu \mathrm{m}]$, channel $10[12 \mu \mathrm{m}]$

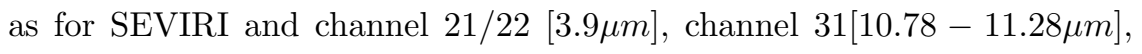
channel $32[11.77-12.27 \mu \mathrm{m}]$ as for MODIS); $L_{\lambda, T}$ is the spectral radiance of the surface according to the Planck's law, at temperature $T$ and wavelength $\lambda$; pb is the portion of pixel at background temperature $\mathrm{Tb}, \mathrm{pc}$ is the portion of pixel at crust temperature Tc and ph is the portion of pixel with active lava at temperature Th. The unknown variables in this system are: pb, pc and Tc since we fix Th to $1363 \mathrm{~K}$ the extrusion temperature for Etna lavas and estimate $\mathrm{Tb}$ from the near lava-free pixels. Once we found the feasible solutions of the nonlinear system, we can compute the thermal flux for the 
high temperature component according to Stefan-Boltzmann law.

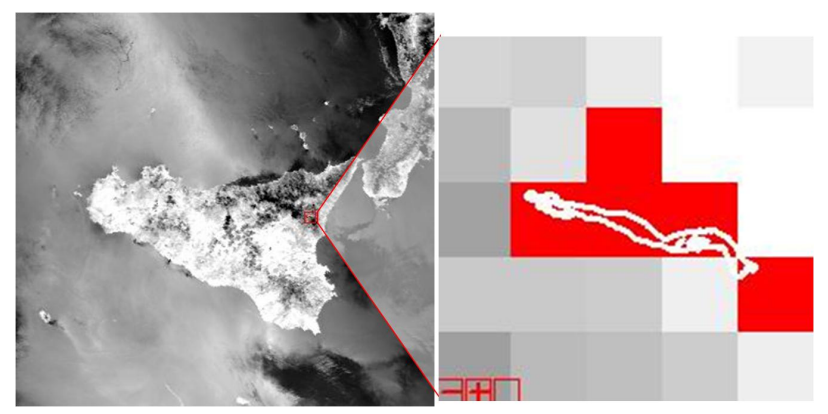

Fig. 1. HotSpot detection for MODIS data.

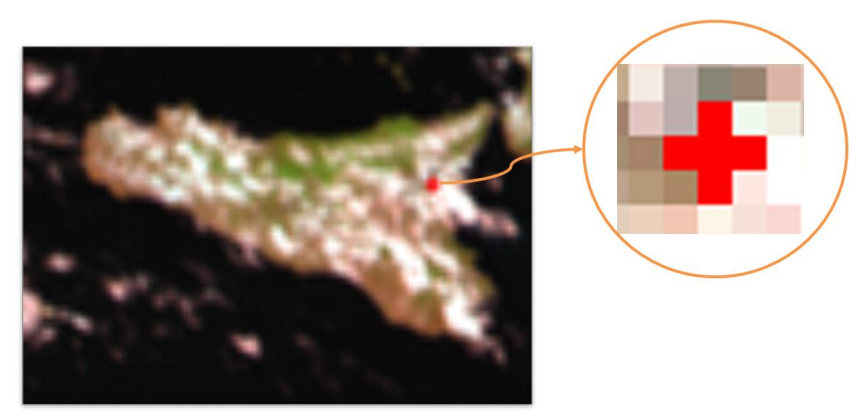

Fig. 2. HotSpot detection for SEVIRI data.

[3] and [10] showed that the total thermal flux measured using satellite infrared data can be converted to time-averaged discharge rate (E) at the time the image data were collected, by using:

$$
E=\frac{Q_{T O T}}{\rho\left(C_{P} \Delta T+C_{L} \Delta \phi\right)}
$$

where $Q_{T O T}$ is the total thermal flux, $\rho$ is the lava density, $C_{P}$ is the specific heat capacity, $\Delta T$ is the eruption temperature of minus solidus temperature, $C_{L}$ is the latent heat of crystallization, and $\Delta \phi$ is the volume percent of crystals that form while cooling through $\Delta T$. 


\section{A case study: Etna 2008 eruption}

Etna's 2008 eruption provided the opportunity to verify our model's ability to predict the path of lava flows while the event was ongoing and to produce different scenarios as eruptive conditions changed. A new eruption started on the morning of 13 May 2008 and it still going on. After a seismic swarm of more than 200 earthquakes and significant ground deformation a fissure erupted in the summit area immediately to the east of Etna's summit craters. On the afternoon of the same day, a new eruptive fissure opened with a number of vents displaying Strombolian activity and emission of lava flows toward the Valle del Bove (a wide depression that cuts the eastern flank of the volcanic edifice). An helicopter survey carried out on 14 May at 13:00 showed the two eruptive fissures: a first one opened on the east of the summit craters (3000 m asl) spreading along North-South direction and a second fissure started from the east flank of South-East Crater summit cone of Mt Etna (2900-2500 m asl) spreading with ENEWSW orientation toward the Valle del Bove. During the following 24 hours the lava traveled approximately $6 \mathrm{~km}$ to the east, but thereafter its advance slowed and stopped, the most distant lava fronts stagnating about 3 $\mathrm{km}$ from the nearest village, Milo. Between 16 and 18 May ash emissions became more frequent and produced small but spectacular clouds, whereas the rate of lava emission showed a gradual diminution. During late May and the first week of June, the activity continued at low levels, with lava flows advancing only a few hundred meters from the vents as of 4 June. Four days later, on 8 June, there was a considerable increase in the vigor of Strombolian activity and lava output rate. During the following week, lava flows advanced up to $5 \mathrm{~km}$ from the source vents. Volcanic thermal anomalies have been observed almost continuously over the same Mt Etna flank in accordance with the occurrence of the lava effusion. A significant increase in the number of hotspots detected, with an evident increase even in their relative intensity, was instead recognized in the late evening of 13 May in accordance with the opening of the new eruptive fissure on NE side of SE Crater. Starting from 14 May several hotspots of high intensity have been flagged over the target area, indicating the clear presence of a lava flow. The time-varying effusion rate during 14 May-16 July was estimated from MODIS and SEVIRI data allowing a detailed chronology of lava flow emplacement (refresh rates: 15 minutes). The lava discharge reached a peak during the first days of the eruption; on 23 May the discharge rate decreased keeping medium-low values of about $1-2 m^{3} s^{-1}$ until 8 June. After 8 June, the effusion rate increased showing scattered values; anyway 
this behavior could be due to an increasing of strombolian activity registered during this period. By integrating minimum and maximum effusion rate values we computed the cumulative curves of erupted lava, finding that the erupted volume is constrained between 8 and 20 million cubic meters. The estimated time-varying effusion rate was also tested, with good results, giving it as input to the numerical simulation of the lava flow path. The good agreement between simulated and mapped flow areas indicates that model-based inundation predictions, driven by time-varying discharge rate data, provide an excellent means for assessing the hazard posed by on-going effusive eruptions.

\section{References}

1. Crisp, J., Baloga, S., Journal of Geophysical Research, 95:1255-1270,(1990).

2. Harris, A.J.L., Dehn, J., Calvari, S., Bulletin of Volcanology, doi:10.1007/s00445-007-0120-y,(2007).

3. Harris, A. J. L., Butterworth, A. L., Carlton, R. W., Downley, I., Miller, P., Navarro, P., and Rothery, D. A., Bulletin of Volcanology, 59, pp 49-64,(1997).

4. Herault, A., Vicari, A., Ciraudo, A., and Del Negro, C., Computers $\&$ Geosciences, doi:10.1016/j.cageo.2007.10.008,(2007).

5. Higgins, J., Harris, A.J.L., Computers \& Geosciences, 23(6):627-645,(1997).

6. Hirn, B., Di Bartola, C., Laneve, G., Cadau, E. and Ferrucci, F.,IEEE International Geoscience 85 Remote Sensing Symposium July 6-11, Boston, Massachusetts, U.S.A.,(2008).

7. Laneve, G., Cadau, E. G.,Geoscience and Remote Sensing Symposium, IGARSS, doi 10.1109/IGARSS.2007.4423337, pp 2447-2450,(2007).

8. Pieri, D.C., Baloga, S.M.,J Volcanol Geotherm Res, 30:29-45,(1986).

9. Vicari, A., Ganci, G., Ciraudo, A., Herault, A., Corviello, I., Lacava, T., Marchese, F., Del Negro, C., Pergola, N., Tramutoli, V., Use of Remote Sensing Techniques for Monitoring Volcanoes and Seismogenic Areas, USEReST,(2008).

10. Wright, R., Blake, S., Harris, A., Rothery, D.,Earth and Planetary Science Letters, 192:223-233,(2001). 